\title{
The mechanics of tumour formation
}

When tumours arise in an epithelial tissue, they disrupt the normal architecture of that tissue. However, very little is known about the mechanical processes that govern such disruption. In a study published in Nature, Messal, Alt et al. report the use of a new imaging technique that has shed light on the morphology of developing tumours within pancreatic ducts.

The authors initially developed an imaging technique that they called FLASH (fast light-microscopic analysis of antibody-stained whole organs). FLASH allows the visualization of whole organs (that have been removed from mice and fixed) in three dimensions and the examination of tissue architecture both overall and at the single-cell level.

Tumorigenesis and disruption of epithelial architecture in the pancreas were triggered using conditional expression of oncogenic $\operatorname{Kras}\left(\operatorname{Kras}^{\mathrm{G} 12 \mathrm{D}}\right)$ with deletion of the tumour suppressor Fbxw 7 in adult duct cells driven by either Krt19 or $H n f 1 b$. In both models, two distinct morphologies were observed using FLASH. Some lesions grew basally, away from the duct lumen (termed exophytic lesions), and others grew apically, towards the duct lumen (endophytic lesions). Exophytic lesions formed globular structures, leading to ductal neoplasia formation, whereas endophytic lesions formed papillary structures leading to intratubular neoplasia.

Similar structures were observed in mice lacking Trp53 instead of Fbxw 7 in the pancreas, indicating that these lesion morphologies are not specific to the combination of $\mathrm{Kras}^{\mathrm{G} 12 \mathrm{D}}$ and $F b x w 7$ loss. Furthermore, the endophytic and exophytic morphology was also observed in patients with pancreatic ductal adenocarcinoma.

Looking in more detail at the cellular morphology and microenvironment around these lesions revealed that exophytic lesions recruited more cancerassociated fibroblasts and had more cells with a mesenchymal morphology compared with endophytic lesions.

As cellular mechanobiology underlies tissue and tumour morphology, the authors investigated the role of the actomyosin cortex in pancreatic tumour development. F-actin and phosphorylated myosin light chain 2 (pMLC2) levels were higher at the apical side of non-transformed cells both in vivo and in organoid cultures, but upon transformation this polarized gradient was reduced. Further analysis of organoids indicated that oncogenic KRAS signalling was crucial for the redistribution of pMLC2.

The authors then built a computational model of pancreatic duct formation. Their experimental observations on the levels of pMLC2 and cell numbers over time were inputted into the model to mimic transformation and subsequent proliferation. Morphological changes akin to exophytic and endophytic lesions were predicted by this modelling, and the main parameter predicting lesion morphology was duct diameter and thus local epithelial curvature. This was confirmed experimentally: endophytic lesions formed in ducts with a larger diameter $(>17 \mu \mathrm{m})$, and exophytic lesions formed in smaller ducts (diameter $<17 \mu \mathrm{m}$ ).

Guillaume Salbreux, one of the corresponding authors of the study, explained that, "Both the data and our models indicate that the two different mechanisms of tumour growth are purely down to the innate physics of the system. Like most cancers, ductal pancreatic cancer starts with a single defective cell that starts dividing. We found that very quickly, when there are only a few cells, the tumour has already started to grow either inwards or outwards depending on duct diameter."

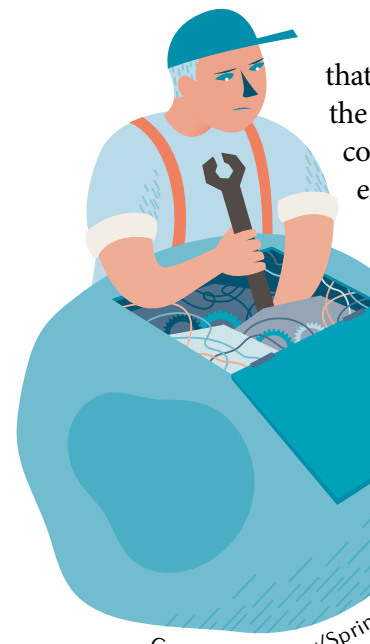

The authors' model also predicted that hyperproliferation alone in the absence of changes in pMLC2 could result in exophytic but not endophytic deformation. Data

from mice in which duct cells

hyperproliferated but were not transformed were consistent with this prediction, as the mice did not have changes in pMLC2 distribution and had mild expansion of the duct lumen. This argues that only endophytic lesion formation requires Credit:Lara Crow/Spin the observed changes in apical-basal tension.

Finally, the authors addressed whether the connections between lesion shape and ductal diameter applied to other tissues. One of the mouse models with $\mathrm{Kras}^{\mathrm{G} 12 \mathrm{D}}$ expression and Fbxw7 loss also developed neoplastic lesions in lung airways and liver ducts in which there were similar changes in pMLC2 distribution. In the liver, lesion growth was exophytic or endophytic, dependent on duct diameter. The lung airways are all $>30 \mu \mathrm{m}$ in diameter, and in the airways only endophytic lesions were observed, as would be predicted by the authors' modelling and observations.

Axel Behrens, another corresponding author of the study, noted that, "Now that we know pancreatic cancer can develop in these two different ways, we can start looking at whether one is likely to be more aggressive or spread in a different way. Many years from now, this could lead to improved diagnostic or treatment options."

In addition to looking at the potential implications for tumour aggressiveness, further studies will need to establish how universal these principles are in other epithelial tissues transformed by different oncogenes. The techniques developed in this study should prove to be useful tools for such research.

Sarah Seton-Rogers parameter predicting lesion morphology was duct diameter 5 